\title{
Long-Range BOTDA Sensing Using Optical Pulse Coding and Single Source Bi-directional Distributed Raman Amplification
}

\author{
Mohammad Taki, Marcelo A. Soto, Fabrizio Di Pasquale, and Gabriele Bolognini* \\ Scuola Superiore Sant'Anna \\ Via G. Moruzzi 1, 56124 Pisa, Italy \\ *e-mail: g.bolognini@sssup.it
}

\begin{abstract}
In this paper we present a Brillouin optical-time domain analysis (BOTDA) sensor combining optimized optical pulse coding with return-to-zero (RZ) modulation format and first-order bi-directional Raman amplification, using a single, shared, high relative-intensity-noise (RIN) pump source. We show that coding provides great benefits in sensing performance, even when employing noisy high-RIN Raman fiber-lasers degrading single-pulse BOTDA-schemes. Combining pulse coding with the use of a single high-power source for Raman amplification allows us to achieve distributed sensing over $120 \mathrm{~km}$ SMF, achieving $2.3 \mathrm{~m}$ spatial resolution with $52 \mu \varepsilon / 2.6^{\circ} \mathrm{C}$ strain/temperature resolutions.
\end{abstract}

\section{INTRODUCTION}

Since the first demonstration of the strain dependence of Brillouin frequency shift in an optical fiber [1], distributed temperature and strain sensors based on stimulated Brillouin scattering (SBS) have become very attractive for industrial applications in strategic sectors. Among distributed optical fiber sensors based on SBS, Brillouin optical time-domain analysis (BOTDA) has been attracting a great interest thanks to its capabilities to perform accurate distributed temperature and strain measurements, with meter and sub-meter scale spatial resolution, over tens of $\mathrm{km}$ of single-mode fibers (SMF). Recently, several techniques have been combined together with BOTDA in order to enhance the sensing performance.

While some techniques have been proposed to achieve high spatial resolution sensing [2], other ones have been focused on extending the sensing range while keeping an accurate temperature/strain resolution, for instance by the use of pulse coding [3] or the use of distributed Raman amplification [4]. Recent studies proposed the combined use of Raman amplification and optical pulse coding to achieve BOTDA with very long measurement ranges and more accurate temperature/strain resolutions [5]. However, arising detrimental issues related to the high relative-intensity noise (RIN) in Raman pumps and to non-optimized pulse coding conditions have given rise to strongly degraded sensor performance.

In this paper we combine the use of optimized pulse coding with return-to-zero (RZ) modulation format [6] and bi- directional distributed Raman amplification [4]. We employ only one high-RIN fiber Raman laser, whose light is shared for both co- and counter- propagating Raman pumping, allowing us to evaluate the impact of optimized pulse coding under the effects of high noise conditions resulting from the high-RIN Raman pumps. The experimental results show that the proposed optimized technique allows for a significant enhancement in the performance of Raman-assisted BOTDA sensors (using high-RIN Raman pumps) with respect to similar works [5].

BOTDA sensor experiments have been carried out by using parameters (i.e. input power values) which have been optimized through numerical simulations as in [7]. Experimental results demonstrate the feasibility to perform distributed temperature/strain measurements over $120 \mathrm{~km}$ SMF using first order bi-directional Raman amplification with a single high-RIN laser source and 127-bit Simplex coding using return-to-zero (RZ) modulation format, achieving $2.3 \mathrm{~m}$ spatial resolution with $52 \mu \varepsilon / 2.6^{\circ} \mathrm{C}$ strain/temperature resolutions

\section{THEORY}

The SBS process is a nonlinear effect in which two counter-propagating optical waves, so called probe and pump, at different frequencies, interact with an acoustic wave along the sensing fiber. This interaction gives rise to optical amplification (or depletion) of the probe intensity. The maximum amplification (or depletion) is reached when the frequency difference between pump and probe is equal to the acoustic wave frequency, which is called Brillouin frequency shift (BFS). Equation (1) shows that the BFS is proportional to the acoustic velocity and the refractive index of the fiber [8].

Thus, the sensing of physical parameters, such as temperature and strain, which impact on the aforementioned quantities can be potentially carried out by simply measuring the BFS:

$$
v_{B}=\frac{2 n \cdot v_{a}}{\lambda}
$$


where $n$ is the refractive index of the optical fiber, $v_{a}$ is the acoustic velocity along the fiber, and $\lambda$ is the pump wavelength. Brillouin-based distributed sensing is obtained by measuring the BFS along the fiber. The most widely technique used to determine the BFS at each fiber location is the socalled BOTDA method, in which a pulsed beam interacts with a counter-propagating continuous-wave probe signal (assuming Brillouin gain configuration) through an acoustic wave generated in the fiber by SBS process. This technique provides a spatial resolution which is given by the interaction length between pump and probe signals. By sweeping the frequency difference between pulsed and probe signals around the BFS, the Brillouin gain spectrum can be measured at every fiber position, and then the local BFS can be obtained by simply fitting a Lorentzian curve to the measured spectrum.

In normal operating conditions, the energy transfer due to SBS amplification in a BOTDA sensor is very small, and therefore, the intensity variations in the probe signal at the photo-receiver can be linearized [3] as follows

$$
\Delta I_{C W}(t, \Delta v) \propto \int_{v_{g} \cdot t / 2}^{v_{g} \cdot t / 2+\Delta z} g_{B}\left(z^{\prime}, \Delta v\right) I_{P}\left(z^{\prime}, \Delta v\right) d z^{\prime},
$$

where $v_{g}$ is the group velocity, $\Delta z$ is the spatial resolution, $\Delta v$ is the pump-probe optical frequency separation, $g_{B}\left(z^{\prime}, \Delta v\right)$ is the Brillouin gain coefficient and $I_{P}\left(z^{\prime}, \Delta v\right)$ is the pump intensity. The Brillouin gain spectrum (BGS) is then reconstructed by tuning the pump-probe frequency difference $\Delta v$, and the BFS is correspondingly obtained, providing information about the local fiber temperature and/or strain. The maximum usable pump and probe power levels are limited by the onset of nonlinear effects and pump depletion that induces nonlocal probe gain effects. Equation (2) points out a well-known trade-off between measurement SNR (and hence, measurand resolution) and the maximum sensing distance (considering a given spatial resolution). A significant SNR improvement can be provided by optical pulse coding schemes [3], in which the single-pulsed pump is replaced by a sequence of pulses following i.e. Simplex coding scheme. Note that this is only possible when linear SBS interaction can be assumed, i.e. when the Brillouin pump can be considered undepleted and the interaction among pulses is negligible. Actually, to minimize the Brillouin interaction among pulses, and hence, possible distortions in the measured BGS, pulses with RZ modulation format have to be used [6]. In addition, bi-directional distributed Raman amplification can also be employed to further enhance the sensing performance; thus two CW Raman pumps can be coupled into both fiber ends, leading to distributed amplification of both Brillouin pump and probe lights [4].

In this paper we show that combining pulse coding with RZ modulation format and optimized distributed bidirectional Raman amplification (based on a single highpower fiber Raman laser at $1450 \mathrm{~nm}$ ) provides an effective technique to overcome the above-mentioned trade-off, enhancing the attained SNR. Note that the enhanced SNR provided by pulse coding, with respect to a single pulse, allows us to relax the requirement on the pump RIN levels and to use a single high-power and high-RIN fiber Raman laser, whose power can be split for both co- and counterpropagating pumping.

\section{EXPERIMENTAL SETUP}

Figure 1 shows the experimental setup of the implemented Simplex-coded BOTDA sensor employing a single fiber Raman laser at $1450 \mathrm{~nm}$ for bidirectional distributed Raman amplification. The coded Brillouin pump and probe signals are generated through the same laser source, given by a distributed-feedback (DFB) laser $(1550 \mathrm{~nm})$, whose optical power is split into two branches using a $3-\mathrm{dB}$ optical coupler $(\mathrm{OC} 1)$.

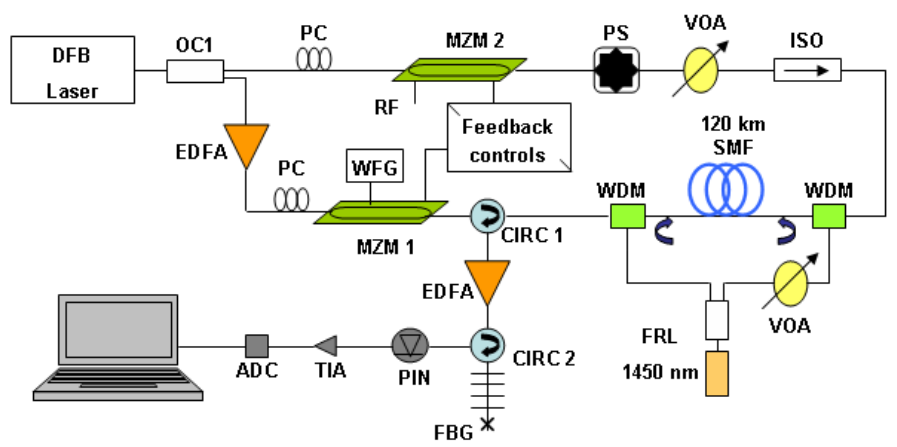

Figure 1. Experimental setup

In the pump branch, the CW-power from the DFB is amplified through an Erbium-doped fiber amplifier (EDFA). The stream of coded optical pulses is generated through a Mach-Zehnder modulator (MZM1) with high extinction ratio, which is driven by a programmable waveform generator (WFG) to provide 127-bit Simplex-coded pulses with a pulse duration of $20 \mathrm{~ns}$ and a duty cycle of $25 \%$ (i.e. a bit slot of $80 \mathrm{~ns}$ ), allowing for a spatial resolution of $2 \mathrm{~m}$. Note that the location of the MZM1 after the EDFA and the use of a polarization-controller (PC) allows us to avoid codeword distortion and ASE noise due to dynamic EDFA operation with optical bursts.

On the other hand, the CW-light in the probe branch is modulated by another MZM (MZM2), preceded by a PC for polarization adjustment, and driven by a programmable RF signal generator in order to generate two carrier-suppressed sidebands with controllable frequency separation. Optimized and stable carrier suppression, as well as stable high extinction ratio coded pump pulses, have been achieved by properly setting the DC bias of the respective MZMs and implementing two automated feedback schemes (also employing optical tap couplers not shown in Fig. 1) to lock the bias of each MZM to the carrier minimum condition and to the minimum inter-pulse light condition, respectively. A variable optical attenuator (VOA) is employed to suitably adjust the probe power coupled into the fiber. The probe sidebands are depolarized 
using a polarization scrambler (PS) in order to reduce polarization issues in the SBS gain.

The Brillouin Simplex-coded pump and the probe are launched into and extracted from $120 \mathrm{~km}$ of SMF (composed of two fiber spools of $60 \mathrm{~km}$-long with similar BFS at room temperature) through two WDM combiners, which are also used to couple the split Raman pump into the co- and counterpropagating directions of the sensing fiber. The used Raman pump is characterized by high power and high RIN level, of the order of $-95 \mathrm{~dB} / \mathrm{Hz}$ (up to several hundred $\mathrm{MHz}$ ). An optical splitter and a variable optical attenuator allow us to regulate the amount of pump power which is coupled into the sensing fiber along forward and backward directions, as shown in Figure 1.

The optimized pumps and probe values have been identified by numerical simulations [7] in order to provide enough optical SNR (OSNR), avoiding at the same time nonlinearities and pump depletion effects. In particular these values are: i) $10 \mathrm{dBm}$ for the input Brillouin pump, ii) $400 \mathrm{~mW}$ for the forward-propagating Raman pump, iii) $20 \mathrm{dBm}$ for the probe power, and iv) $300 \mathrm{~mW}$ for the backward-propagating Raman pump. Note that such optimized power levels ensure an OSNR of $\sim 40 \mathrm{~dB}(0.1 \mathrm{~nm}$ bandwidth), resulting in a minimum Brillouin pump-probe power difference of $\sim 17.5 \mathrm{~dB}$ along the whole sensing fiber, and avoiding then pump depletion. At the receiver side, after an optical circulator (CIRC1), a linear EDFA is used as a preamplifier, followed by a second optical circulator (CIRC2) and a narrowband fiber Bragg grating (FBG, with a bandwidth of $48 \mathrm{pm}$ ), allowing us to select the Brillouin Stokes component, while filtering out the Brillouin anti-Stokes, Rayleigh, residual carrier and ASE noise from the EDFA. The photo-detection and data acquisition are carried out by PIN photodiode (125-MHz bandwidth), followed by a transimpedance amplifier (TIA), voltage amplification stages (VGA), an analog-to-digital converter (ADC) and a computer for data processing.

\section{EXPERIMENTAL RESULTS}

In order to study the benefits of the pulse coding in presence of high RIN Raman pumps, we have compared the measured BOTDA traces, obtained at maximum gain (Brillouin pump-probe frequency difference equal to the average BFS of the second fiber spool) for single pulse and Simplex coding, as illustrated in Fig. 2. In this figure we can observe the evident SNR improvement provided by the use of Simplex coding in presence of high-RIN Raman pumping. By comparing the SNR of both traces, the average SNR enhancement has been found to be $\sim 7.4 \mathrm{~dB}$.

BOTDA traces have been measured within a spectral range of $120 \mathrm{MHz}$. After decoding the traces, the local BGS has been fitted with a Lorentzian curve at every fiber position. Figure 3 shows the decoded BGS measured with the optimized system parameters.

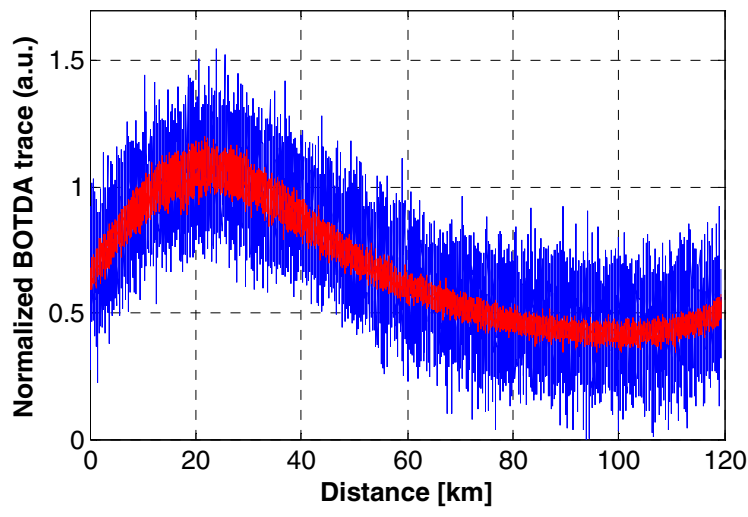

Figure 2. BOTDA traces measured at maximum gain frequency

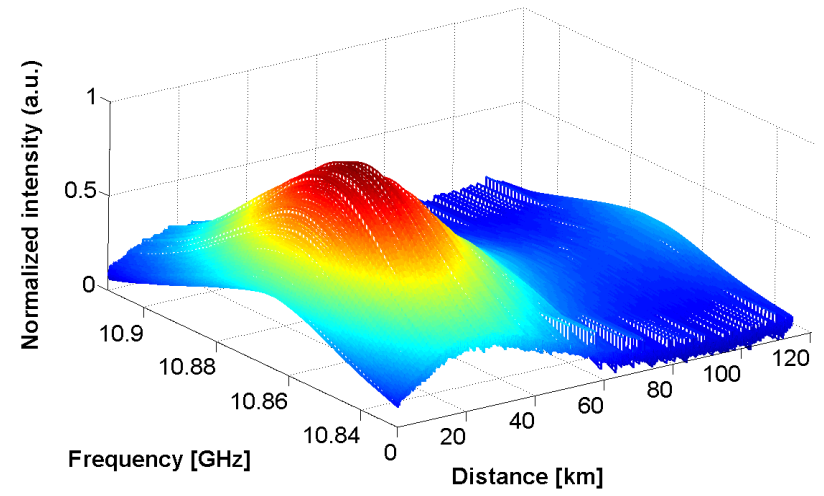

Figure 3. BGS along the sensing fiber with coded Raman-assisted BOTDA

In order to calculate the frequency accuracy along the whole sensing fiber of the coded Raman-assisted BOTDA, the BFS parameter has been calculated from the measured BGS and is shown in Fig. 4. By calculating the standard deviation of the BFS, we can then obtain the frequency resolution of the sensor, which was found to be $\sim 2.6 \mathrm{MHz}$ at around $100 \mathrm{~km}$ distance (where the lowest SNR value is observed), thus leading to temperature and strain resolutions better than $52 \mu \varepsilon / 2.6^{\circ} \mathrm{C}$ over $120 \mathrm{~km}$ fiber length. Effects of pump depletion (even in presence of long Simplex-coded pump pulse sequences) can be considered negligible in our experiments, as expected by the theoretical optimization of the used input power levels, and as confirmed by the absence of any significant deviation in the BGS and BFS trace (see Figs. 3-4).

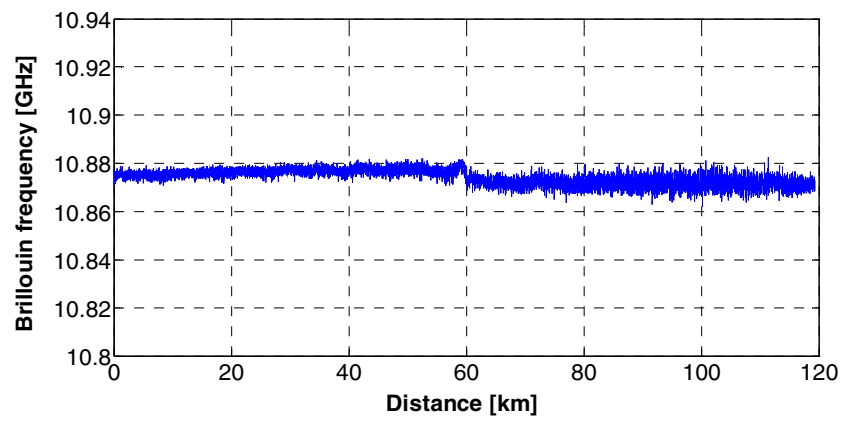

Figure 4. BFS vs distance along the sensing fiber 
Figure 5 shows the temperature resolution over the $120 \mathrm{~km}$ sensing fiber. As we can see, the temperature resolution calculated over the first $40 \mathrm{~km}$ was less than $\sim 1.5{ }^{\circ} \mathrm{C}$, thanks to the effects of the forward-propagating Raman pump, which strongly amplifies the Brillouin pump thus enhancing the SBS interaction; the resolution degrades then up to $\sim 2.6^{\circ} \mathrm{C}$ (around $100 \mathrm{~km}$ distance) due to attenuation and low SNR. Note that the amplification resulting from the backward-propagating Raman pump increases the Brillouin interaction near the far fiber end, leading to an enhanced SNR and an improved temperature resolution at very long distances.

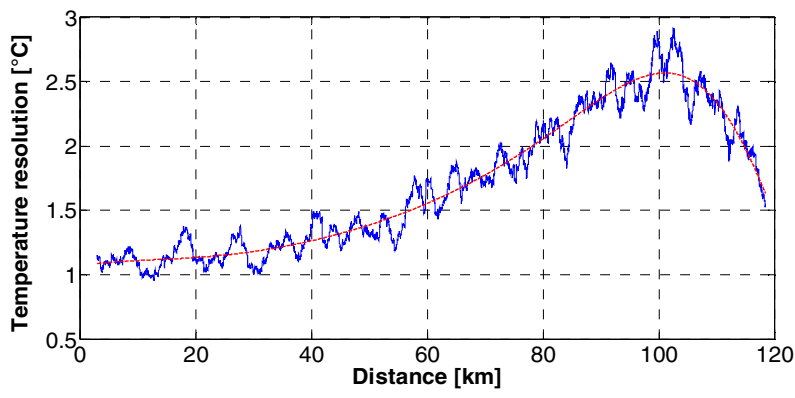

Figure 5. Temperature resolution vs distance along the sensing fiber

In order to calculate the spatial resolution of the sensor, the temperature of a short fiber spool (15 m length), near the far fiber end $(119.93 \mathrm{~km})$, has been increased up to $50{ }^{\circ} \mathrm{C}$ in a temperature controlled chamber, while the rest of fiber has been kept at room temperature $\left(28{ }^{\circ} \mathrm{C}\right)$. Figure 6 shows the BGS measured at the end of the fiber, where the frequency shift in the local BGS induced by heating can be clearly observed.

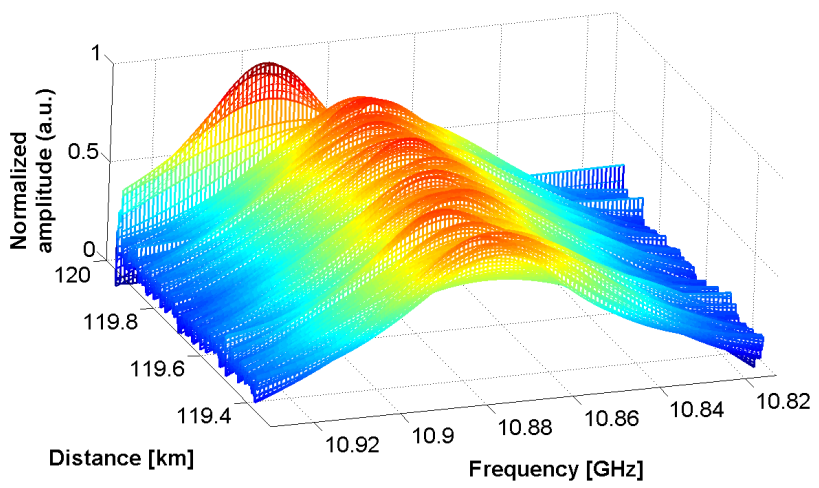

Figure 6. Measured BGS near the fiber end

The temperature profile (obtained from the BFS parameter) near the far fiber-end is reported in Fig. 7; the figure also shows the ability to effectively detect temperature changes with a spatial resolution which has been measured equal to $\sim 2.3 \mathrm{~m}$ (as the $10 \%-90 \%$ step-response for an abrupt temperature variation).

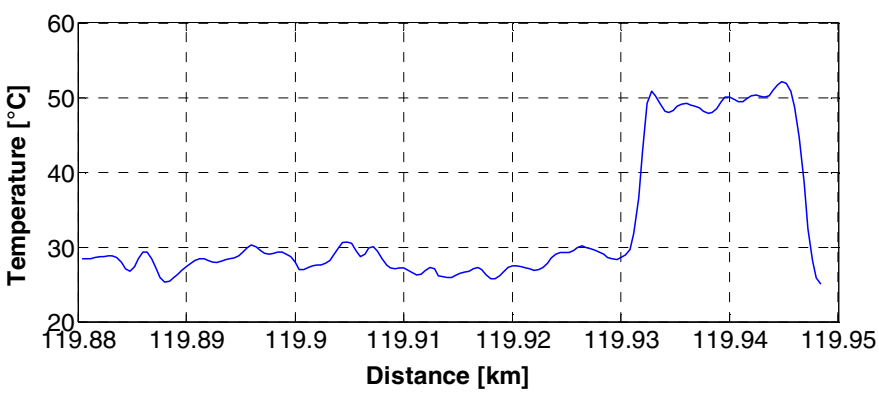

Figure 7. Temperature profile near the fiber end

\section{CONCLUSIONS}

In conclusion we have implemented a BOTDA sensor combining 127-bit optical coding and first order bi-directional Raman amplification using one single shared high RIN source. Optical coding has been shown to provide a great benefit in sensing performance even when employing noisy high-RIN Raman sources strongly degrading single-pulse BOTDA systems. This has actually allowed us to use one single shared high-power source for co- ad counter-propagating Raman amplification, and to attain distributed sensing over $120 \mathrm{~km}$ of $\mathrm{SMF}$, achieving a spatial resolution of $2.3 \mathrm{~m}$ with $52 \mu \varepsilon / 2.6^{\circ} \mathrm{C}$ strain/temperature resolutions.

\section{REFERENCES}

[1] T. Horiguchi, T. Kurashima, and M. Tateda, "Tensile strain dependence of Brillouin frequency shift in silica optical fibers," IEEE Photon. Tech. Lett., vol. 1, no. 5, pp. 107-108, 1989.

[2] W. Li, X. Bao, Y. Li, and L. Chen, "Differential pulse-width pair BOTDA for high spatial resolution sensing," Opt. Express, vol. 16, no. 26, pp. 21616-21625, 2008.

[3] M. A. Soto, G. Bolognini , F. Di Pasquale, and L. Thévenaz, "Simplexcoded BOTDA fiber sensor with $1 \mathrm{~m}$ spatial resolution over a $50 \mathrm{~km}$ range," Opt. Lett., vol. 35, no. 2, pp. 259-261, 2010.

[4] F. Rodriguez-Barrios et al. "Distributed Brilouin fiber sensor assisted by first-order Raman amplification," J. Light. Tech., vol. 28, no. 15, pp. 2162-2172, 2010

[5] Xin-Hong Jia et al. "Experimental demonstartion on $2.5 \mathrm{~m}$ spatial resolution and $1{ }^{\circ} \mathrm{C}$ temperature uncertainty over long-range-distance BOTDA with combined Raman amplification and optical pulse coding," IEEE Photon. Tech. Lett. vol. 23, no. 7, pp. 435 - 437, 2011.

[6] M. A. Soto, G. Bolognini, and F. Di Pasquale, "Analysis of pulse modulation format in coded BOTDA sensors," Opt. Express, vol.18, no.14,pp. 14878-14892, 2010.

[7] M. A. Soto, G. Bolognini, and F. Di Pasquale, "Optimization of longrange BOTDA sensors with high resolution using first-order bidirectional Raman amplification," Opt. Express, vol. 19, no. 5, pp. 4444-4457, 2011

[8] M. Niklès, L. Thèvenaz, and P. A. Robert, "Brillouin gain spectrum characterization in single-mode optical fibers," J. Light. Tech., vol. 15, no. 10, 1997. 